334

\section{NEURODEVELOPMENTAL OUTCOME OF PREMATURE INFANTS TREATED WITH INHALED NITRIC OXIDE}

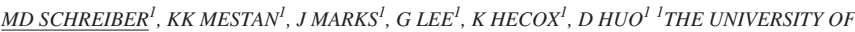
CHICAGO (USA)

In a randomized, placebo-controlled trial of 207 premature infants with respiratory distress syndrome, we previously reported inhaled nitric oxide (iNO) decreased the incidence of chronic lung disease (CLD) and death (Schreiber et al., NEJM, 2003). The study also found that iNO decreased severe intraventricular hemorrhage/perventricular leukomalacia (IVH/PVL). To evaluate the long-term neurodevelopmental outcomes of these premature infants treated with iNO, we conducted a prospective follow-up of the original cohort (BW $983378 \mathrm{~g}$, GA $27.02 .7 \mathrm{wks}$ ) to two years corrected age. The Bayley Scales of Infant Development and neurologic examinations were performed by examiner blinded to the original treatment assignment. Abnormal neurodevelopmental outcome was defined a having: A diagnosis of cerebral palsy, bilateral blindness or hearing loss, and/or an MDI or PDI $<70$. A total of 138 infants (82\% of survivors) were followed. Compared with placebo-treated infants, patients treated with iNO had a significantly lower risk of an abnormal neurodevelopmental outcome (relative risk $0.53 ; 95 \%$ confidence interval, $0.33-0.87 ; \mathrm{P}=0.01$ ). After adjustment for $\mathrm{CLD}$, the risk of abnormal outcome was still significantly reduced in the iNO group (relative risk, $0.59 ; 95 \%$ confidence interval, $0.36-0.95 ; \mathrm{P}=0.03$ ). Similarly, the risk remained reduced even after adjustmen for severe IVH/PVL (relative risk, $0.55 ; 95 \%$ confidence interval $0.34-0.89 ; \mathrm{P}=0.01$ ). The iNO group had approximately one half the risk of cognitive impairment $(\mathrm{MDI}<70)$ and/or psychomotor impairment $(\mathrm{PDI}<70)$ compared with the placebo group (relative risk, $0.55 ; 95 \%$ confidence interval, $0.33-0.93 ; \mathrm{P}=0.03$ ). This difference was due primarily to a decrease in cognitive impairment in the iNO group (relative risk, $0.53 ; 95 \%$ confidence interval, $0.29-0.94 ; \mathrm{P}=0.03$ ) but not in psychomoto impairment (relative risk, $0.73 ; 95 \%$ confidence interval, $0.33-1.61 ; \mathrm{P}=0.48$ ). In conclusion, premature infants treated with iNO in the first week of life had a significantly lower risk of having an abnormal neurodevelopmental outcome at two years of age.

\section{5}

\section{LONG-TERM CONTINUOUS EEG-MONITORING IN VERY PREMATURE CHILDREN}

EM SCHUMACHER, H SJOHOLM, T STIRIS ${ }^{1}$ DEPTS OF PAEDIATRICS - NICU AND CLINICAL NEUROPHYSIOLOGY ULLEVAL UNIVERSITY HOSPITAL (NORWAY)

Background: The immature brain is vulnerable for insults the first days of life. It is desirable to have a method for continuous monitoring of the brain in this critical period of time. Unfortunately, available methods like time-compressed amplitude integrated EEG based on only few channels, have definite limitations.

Objective: To devise a method for stable continuous long-term EEG monitoring covering both hemispheres of the brain.

Methods: After consent from the parents and approval from the Ethics Committee thirty premature babies below 30 weeks of gestational age were included within twelve hours after birth. We used a digital EEG system ( NERVUS a/S ) for continuous EEG monitoring during their first three days of life. 10 electrodes were symmetrically placed over both hemispheres leaving space over vertex for ultrasound measurements to be made when needed

Results: Out of 30 patients we succeeded to perform full-length (72 hours) recordings in 28 cases Successful recordings were characterized by stable electrode contacts with impedances within the normal range which were repeatedly checked every third hour. There were no apparent signs that the scalp electrodes disturbed the patients. Digital recordings make it possible to quantify general and focal changes in the brain as well as seizure activity.The predictive value of the EEG changes for later outcome will be established when we compare early EEG patterns to the patients' status at 2 years of age.

Conclusions: We have demonstrated that it is feasible to perform long-term continuous EEC monitoring in very premature children. This should be of importance in order to detect general or focal brain dysfunction where rapid intervention is advantageous.

\section{6}

TROPONIN SERUM LEVELS AND ECHOCARDIOGRAPHIC LEFT VENTRICULAR EVALUATION IN PRETERM NEWBORNS WITH RESPIRATORY DISTRESS SYNDROME

C MATTIA, P SCIACCA, P. BETTA, M.G. ROMEO, G. DISTEFANO I UNIT OF PEDIATRIC CARDIOLOGY - DEPARTMENT OF PEDIATRICS , ${ }^{2}$ UNIT OF PEDIATRIC CARDIOLOGY DEPARTMENT OF PEDIATRICS, ${ }^{3}$ NICU - DEPARTMENT OF PEDIATRICS, ${ }^{4}$ NICU - DEPART MENT OF PEDIATRICS, ${ }^{5} U$. OF NEONATAL PATHOLOGY (ITALY)

Backgrounds: Perinatal asphyxia is frequently associated with heart impairment. In this study we related troponin serum levels (a cardiac ischaemic index in adults) with left ventricle B-mode functional evaluation in preterm infants with RDS

Methods: We studied 40 hypoxaemic preterm newborns affected by RDS, with gestational age ranging 26-34 w. and weight ranging 890-2400 gr. In all newborns pH, $\mathrm{PaCO} 2, \mathrm{PaO} 2$, HCO3, ABE, SBE, Heart rate, MAP, SaO2 and thoracic radiography, were evaluated at admission to the NICU of University of Catania. Infants were divided in 2 groups: the first with mean $\mathrm{pH} 7,17+-0,05$ and serious RDS needing $\operatorname{IPPV}$ (16 patients); the second with mean $\mathrm{pH} 7,27+-0,03$ and mild RDS not requiring IPPV (24 patients). Troponin determination was made $12 \mathrm{~h}$ after birth together with left ventricle echocardiography evaluation. End diastolic volume, End sistolic volume, Stroke volume (SV), cardiac output (CO) and Ejection Franctio (EF) were studied by using B-mode ellipsoidal method

Results: Mean serum levels of Troponin were significantly higher in the first group with severe RDS $(0,26+-0,161 \mathrm{mcg} / \mathrm{ml})$ than in the second with mild RDS $(0,037+-0,0031 \mathrm{mcg} / \mathrm{ml})$. We demonstrated a significant inverse correlation between Troponin levels and left ventricular functional index that were lower in the first group (SV 0,49+- $12 \mathrm{ml} / \mathrm{b}$; CO $52,5+-14 \mathrm{ml} / \mathrm{min}$; EF $46+-10 \%$ ) than in the second (SV 1,14 +- $32 \mathrm{ml} / \mathrm{b} ; \mathrm{CO} 131,5+-34 \mathrm{ml} / \mathrm{min}$; EF 59,2 +- 8\%). It had been necessary adding inotropic therapy with Dopamine and Dobutamine in 12 patients of the first group

Conclusions: These results suggest that elevated Troponin levels in preterm newborns are related both with hypoxic insult and with cardiac dysfunction. Troponin levels such as echocardiographic b-mode left ventricular evaluation can be helpful to objectively check myocardial function in preterm infants with RDS in order to early recognise heart impairment evaluating the correct use of inotropic therapy.

\section{7}

NEONATAL OUTCOME IN TRIPLET PREGNANCIES

AA ZUPPA ${ }^{l}$, A SCORRANO ${ }^{l}, F$ COTA ${ }^{l}, V$ CALABRESE ${ }^{l}$, I SAVARESE ${ }^{l}, V D^{\prime} A N D R E A^{l}, C$ ROMAGNOLI I INIVERSITÀ CATTOLICA DEL SACRO CUORE (ITALY)

Background In the last 10 years, advances in the induction of ovulation (OI) and assisted reproductive techniques (GIFT, FIVET, ICSI) have resulted in an epidemic of multiple pregnancies Unfortunately, the increase in twin and higher-order gestations has also increased maternal and perinatal risks. Data from recent literature try to point-out differences in obstetric and neonatal outcomes in spontaneus multiple pregnancies versus pregnancies obtained with OS or assisted procreation. The aim of this study is to evaluate and compare spontaneous vs non-spontaneous neonatal outcomes of triplet pregnancies in the Policlinic A. Gemelli, a reference centre for high risk pregnancies.

Methods We studied 24 triplet pregnancies, 6 spontaneous, 7 obtained with OI and 11 obtained with assisted procreation techniques. All 72 newborns were evaluated for: prematurity, neonatal weight, IUGR, birth weight discordance, need for resuscitation at birth, and major neonatal diseases (infective, hematologic, respiratory, cardiovascular, malformative and neurologic).

Results Spontaneous and non-spontaneous triplet pregnancies had similar EG and neonatal weight: EG $33 \pm 1$ weeks vs $33 \pm 2$ weeks, neonatal weight $1705 \pm 256 \mathrm{~g}$ vs $1907 \pm 452$. There were no significative differences in IUGR and birth weight discordance $(>20 \%)$ and in major neonatal diseases. Among non-spontaneous triplets we observed 2 malformative diseases (a polimalformative syndrome and a cri-du-chat syndrome), 2 neurologic diseases (IVH 2 grade), 1 cardiovascular disease (surgically treated PDA) and 1 feto-fetal transfusion.

Conclusions Our results confirm literature data about absence of significative neonatal outcome differences between spontaneous and non-spontaneous triplet pregnancies, but individual clinics and collaborative efforts are needed to extend the number of observations about major neonatal diseases (expecially malformative).

\section{8}

THE UTILITY OF TWO-CHANNEL BEDSIDE EEG MONITORING IN TERM BORN ENCEPHALOPATHIC INFANTS IN RELATION TO CEREBRAL INJURY AS DEFINED BY MR IMAGING

DK SHAH ${ }^{l, 2,3}, S$ LAVERY ${ }^{2,3}, C$ WONG ${ }^{2,3}, K$ JAMSEN ${ }^{l}$, P MCDOUGALLL ${ }^{2}$, TE INDER ${ }^{l, 2,3}{ }^{l}{ }^{M U R}-$ DOCH CHILDRENS RESEARCH INSTITUTE (AUSTRIA), ${ }^{2}$ ROYAL CHILDREN'S AND ROYAL WOMEN'S HOSPITALS (AUSTRIA), ${ }^{3}$ VICTORIA INFANT BRAIN STUDY (AUSTRIA)

Objective: Single channel amplitude-integrated EEG has been shown to be predictive of neurodevelopmental outcome in term infants with hypoxic-ischemic encephalopathy (HIE). We describe the relationship of quantifiable EEG measures, obtained using a two-channel digital bedside EEG monitor, from term newborn infants with encephalopathy and/or seizures to cerebral injury defined qualitatively by MR imaging and also the effect of anticonvulsants on these measures.

Methods: Median values of minimum, mean and maximum EEG amplitude were obtained from 95 term-born encephalopathic infants during a two hour seizure-free period obtained within 72 hours of admission. Ninety-three infants underwent MR imaging with images qualitatively scored for abnormalities of cortex, white matter, deep nuclear grey matter and posterior limb of internal capsule. For seven infants, EEG measures obtained immediately prior to and half an hour after anticonvulsant were compared.

Results: For all infants there was a direct relationship between EEG amplitude measures and MR abnormality scores (MRAS). This relationship was strongest for the minimum amplitude measures $(n=86$, left; $b=-0.86$, SE $0.13, p<0.001$ ). This relationship persisted on sub-group analysis for infants with HIE $(\mathrm{n}=47$, left; $\mathrm{b}=-1.06, \mathrm{SE} 0.19, \mathrm{p}<0.001)$ and after adjusting for the use of anticonvulsants. Using a MRAS cut-off of 7, a minimum amplitude of $4 \mathrm{mcV}$ showed a higher specificity $(85 \%)$ whereas a minimum amplitude of $6 \mathrm{mcV}$ showed a higher sensitivity $(77 \%)$. Anticonvulsant administration produced a mean reduction in minimum amplitude of $0.9 \mathrm{mcV}$. Infants with higher minimum amplitudes prior to anticonvulsant administration showed a larger drop in amplitude after administration.

Conclusion: Bedside EEG measures in term-born encephalopathic infants are related to the severity of cerebral injury as defined by qualitative MRimaging. A minimum amplitude of $4 \mathrm{mcV}$ appears useful in cerebral injury as defined by qualitative MRimaging. A minimum amplitude of $4 \mathrm{mcV}$ appears useful in
predicting outcome whereas $6 \mathrm{mcV}$ may be more useful for screening purposes, such as the enrollment predicting outcome whereas $6 \mathrm{mcV}$ may be
criterion for neuro-protective interventions.
CORRELATION BETWEEN WATER DIFFUSION VALUES AND HISTOPATHOLOGICAL WHITE MATTER CHANGES IN FIXED TISSUE FROM A PRIMATE MODEL OF PREMATURE BIRTH

DK SHAH $\stackrel{1,2}{,}$ HX WANG ${ }^{1,3}, M$ LEOLIGER ${ }^{4}, C D$ KROENKE ${ }^{5}, J J N E I L^{5}, S R^{2} E E S^{4}, T_{E} I N D E R^{1,2,3}$ ${ }^{1}$ VICTORIA INFANT BRAIN STUDY (AUSTRIA), ${ }^{2}$ MURDOCH CHILDRENS RESEARCH INSTITUTE (AUSTRIA), ${ }^{3}$ HOWARD FLOREY INSTITUTE (AUSTRIA), ${ }^{4}$ UNIVERSITY OF MELBOURNE (AUSTRIA), ${ }^{5}$ UNIVERSITY OF WASHINGTON (USA)

Background: Diffusion MR techniques are increasingly utilized in human preterm infants to reflect white matter microstructure and its integrity.

Objective: To assess the relationship of the apparent diffusion coefficient (ADC), from diffusion MR imaging, to the severity of white matter injury and maturation in fixed tissue from a baboon model of premature birth

Methods: Thirty-one baboons were delivered at $125 \mathrm{~d}$ gestational age (GA, equivalent to 28 weeks GA in the human) and were sacrificed between 139 and $160 \mathrm{~d}$ post-conceptional age (PCA). Support included surfactant, ventilator support, inotropic support, TPN, and physiological monitoring. Following sacrifice, the brains were immersion fixed in $10 \%$ formalin. Control data were obtained from 9 animals sacrificed immediately after delivery, from 90 to $160 \mathrm{~d}$. MR data were obtained using a 4.7-T system and DTIs generated using a 2D spin echo pulse sequence. ADC values were analyzed as pairs in the frontal, central, and occipital areas. Forebrain sections were stained with H,E and glial fibrillary acidic protein to assess cerebral injury and gliosis. A white matter abnormality score (WMAS) was formulated using the presence of ventriculomegaly, areas of axonal damage, white matter gliosis and GFAP immunoreactivity in radial glial fibers. Damage was scored throughout the rostral-caudal extent of the brain; scale 0 (no abnormality) to 22 (severe abnormality).

Results: On linear regression there was a direct relationship between the mean ADC and the PCA within the gestational control group $(\mathrm{b}=-0.001, \mathrm{p}=0.01)$. There was also a direct relationship between $\mathrm{ADC}$ and WMAS for each region, which persisted when adjusted for PCA $(b=0.03, p<0.01$ central region).

Conclusions: The association of white matter gliosis and axonal damage with elevated ADC values supports the hypothesis that elevated ADC values, as described in premature infants, reflect white matter injury with associated alterations in white matter microstructure. 